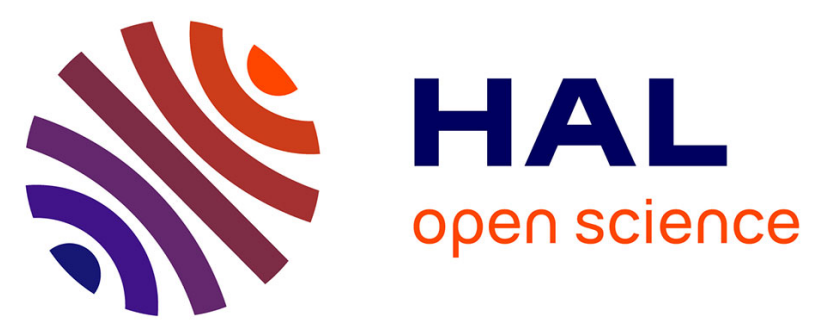

\title{
QUELQUES EXPÉRIENCES SUR L'ÉVOLUTION DES CENTRES COLORÉS DANS LiF SOUS L'INFLUENCE COMBINÉE DE LA TEMPÉRATURE ET DE LA DÉFORMATION PLASTIQUE
}

\author{
J. Serughetti, B. Schaeffer, C. Dupuy, H. Saucier
}

\section{To cite this version:}

J. Serughetti, B. Schaeffer, C. Dupuy, H. Saucier. QUELQUES EXPÉRIENCES SUR L'ÉVOLUTION DES CENTRES COLORÉS DANS LiF SOUS L'INFLUENCE COMBINÉE DE LA TEMPÉRATURE ET DE LA DÉFORMATION PLASTIQUE. Journal de Physique Colloques, 1967, 28 (C4), pp.C4-158-C4-162. 10.1051/jphyscol:1967427 . jpa-00213314

\author{
HAL Id: jpa-00213314 \\ https://hal.science/jpa-00213314
}

Submitted on 1 Jan 1967

HAL is a multi-disciplinary open access archive for the deposit and dissemination of scientific research documents, whether they are published or not. The documents may come from teaching and research institutions in France or abroad, or from public or private research centers.
L'archive ouverte pluridisciplinaire HAL, est destinée au dépôt et à la diffusion de documents scientifiques de niveau recherche, publiés ou non, émanant des établissements d'enseignement et de recherche français ou étrangers, des laboratoires publics ou privés. 


\title{
QUELQUES EXPÉRIENCES SUR L'EVOLUTION DES CENTRES COLORES DANS LiF SOUS L'INFLUENCE COMBINÉE DE LA TEMPERATURE ET DE LA DEFORMATION PLASTIQUE
}

\author{
J. Serughetti, B. Schaefrer, C. H. S. Dupuy et H. Saucier
}

Laboratoire de Minéralogie et Pétrographie de la Faculté des Sciences, 67-Strasbourg

\begin{abstract}
Plastic deformation of $\gamma$ irradiated $\mathrm{LiF}$ single crystals gives, at room temperature, an absorption band at $\lambda=545 \mathrm{~m} \mu\left(N_{2}\right)$ and increases the absolption peak of the $440 \mathrm{~m} \mu$ band $(M)$. These bands are anisotropic. Similar results are found on crystals thermally annealed at $250^{\circ} \mathrm{C}$, however we note that the $525 \mathrm{~m} \mu$ band $\left(N_{1}\right)$ decreases with plastic deformation.

Then, some crystals are deformed at $150^{\circ}, 250^{\circ}$ and $300^{\circ} \mathrm{C}$, we were unable to observe any variations of the absorption band of any given centers; however the $400{ }^{\circ} \mathrm{C}$ thermal annealing of these crystals shows that the deformed zones are darker than the other parts of the crystals. The $F$ and $M$ center " persistence $\gg$ increases linearly with the plastic strain.

Résumé. - La dúformation plastique, à température ambiante, de cristaux de L,iF, itradiés aux rayons $\gamma$, fait apparaittre une bande d'absorption fortement anisotrope pour $\lambda=545 \mathrm{~m} \mu\left(\mathrm{N}_{2}\right)$; elle augmente atussi l'absorption de la bande $M(\lambda==440 \mathrm{~m} \mu)$. Si Fon fait subir une déformation analogue à des cristaux préalablement recuits a $250^{\circ} \mathrm{C}$, on constate les mêmes phenoménes, mais il apparaît cn plus une diminution de la bande d'absorption situce a $525 \mathrm{~m} \mu\left(N_{1}\right)$, diminution qui pourrait étre due à la destruction, par la déformation plastique des centres $N_{1}$.

On a ensuite étudiè au spectrophotomètre des cristaux irradiés préalablemcnt déformés a $150^{\circ}$, $250^{\circ}$ et $300^{\circ} \mathrm{C}$, on ne constate alors aucune variation dans les bandes d'absorption des centres déjà cites. Par contre, si après une telle déformation on effectue un recuit à $400^{\circ} \mathrm{C}$, on remarque que les zones deformeses sont pius colorées que le reste du crjstal.

Lanalyse spectrophotonetrique montre que la persistance des centres $F$ et $M$ augnente avec la déformation, Jeur coefficien! d'absorption variant linéairement avec elle.

L'essai d'interprétation donné pour les déformations à la température ambiante est basé sur la possibilité qu'auraient les dislocations de transformer les centres existants.

Pour la persistance des centres après recuit des cristaux deformés à chaud, on peut supposer quo sous laction du champ de contrainte il se produit une segrégation des défauts : les defauts de type lacunaire migrent dans les parties en compression, ceux du type interstitiel se dirigent vers les parties en tension.
\end{abstract}

Introduction. - L'influence de la déformation plastique sur le mécanisme de formation des centres colorés dans LiF au cours d'irradiation a fait l'objet de recherches par Wiegand, Turner et Smoluchowski [1] et [2], recherches au cours desquelles ces auteurs opéraient à basse température afin de diminuer la complexité des phénomènes.

Au cours d'expériences que nous ayons déjà publiées [3] [4] [5], la déformation plastique était postérieure à l'irradiation ; nous avons pu ainsi étudier son influence sur les centres colorés préexistants.

Dans le présent travail les observations correspondent à des températures progressivement croissantes. Les cristaux utilisés et les méthodes de déformation ont été indiquées antérieurement [6]. Les cristaux ont été irradiés aux rayons $\gamma$ à l'aide d'une source au cobalt 60 , la dose etant d'environ $10^{7} \mathrm{rd}$. Ies spectres d'absorption ont ćté faits avec un spectrophotomètre Beckman DK $2 \mathrm{~A}$. Le montage microspectrophotométrique a dejà été décrit [3]. Les recuits ont une durée uniforme d'une demi-heure ; ils śtaient strivis d'une trempe à l'air ambiant.

I. Cristaux irradiés déformés à température ambiante. - Les premières expériences ont etté conduites à température ambiante. Les cristaux sont déformés en compression jusqu'a l'apparition d'une bande de glissement. L'analyse du spectre d'absorption correspondant à cette bande de glissement se fait grâce à un montage microspectrophotométrique [3].

On constate qu'il apparaît à $545 \mathrm{~m} \mu$ une bande d'absorption ; c'est vraisemblablement la raie $N_{2}$ signalée 
par Kubbo à $540 \mathrm{~m} \mu$ [7]. On observe aussi une augmentation de l'absorption correspondant à la bande $M$ à $440 \mathrm{~m} \mu$. Cette bande est anisotrope. L'absorption est maximale lorsque la direction de vibration de la lumière polarisée transmise est perpendiculaire à l'axe de compression [001]. Il semble que ce soit la mêrne direction que celle correspondant aux centres $N_{2}$. Sur la figure 1 on constate que le dichoïsme manifesté pour la raie $N_{2}$ se retrouve, plus marqué, pour la bande $M$.

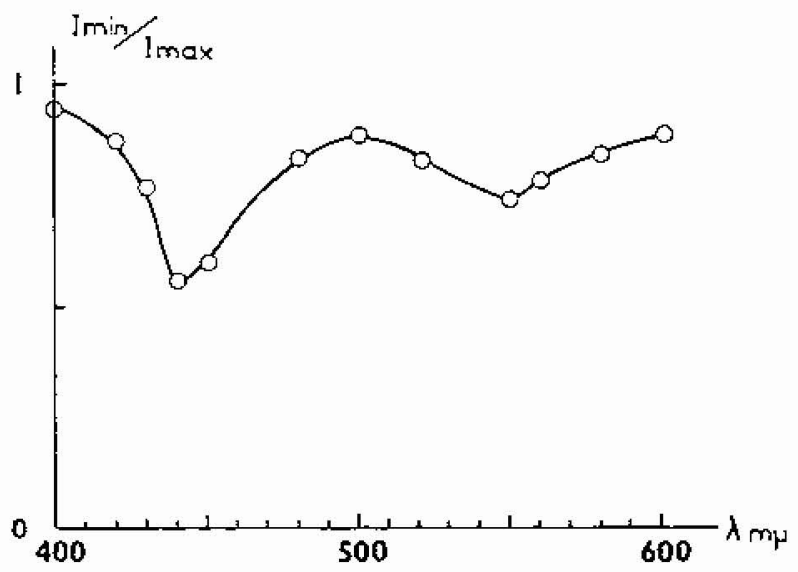

Frg. 1. - Anisotropie observée dans un spectre d'absorption donmé par une bande de glíssement (110) - cristal de LiF ircadié aux rayons $\gamma$ et déforme par compression à température ambiante.

On remarquera d'ailleurs qu'en ćclairant les cristaux irradiés non déformés par de la lumière polarisée rectíligne vibrant suivant une direction [110] on obtient pour la bande $M$ un dichroïsme analogue à celui observé par Van Doorn et Haven [8] dans le chlorure de potassium. Par contre pour cette orientation de la vibration lumineuse, la bande $N_{2}$ ne présente aucun dichroisme.

II. Cristaux irradiés partiellement recuits, déformés à température ambiante. - Lors du recuit des cristaux à $250^{\circ} \mathrm{C}$, on constate un renforcement de la bande $N$, où s'individualisent les bandes $N_{1}$ et $N_{2}$.

Après déformation, on observe les mêmes phénomènes que pour les cristaux non recuits, mais l'absorption diminue dans la zone déformée pour une longueur d'onde de $525 \mathrm{~m} \mu$ (centres $N_{1}$ ). Le dichroisme déjà signalé pour la bande $M$ est visible.

III. Cristaux irradiés déformés à chaud. - Les spectres d'absorption des cristaux irradiés déformés à chaud ont été faits à température ambiante, pour des taux de déformation plastique variant de 0 à $3 \%$. Cette déformation plastique était obtenue par flexion en quatre points.
A la différence de ce que l'on observe pour une déformation à froid, il n'apparaît pas de variation significative du coefficient d'absorption après déformation, pour la bande $M$. Il en est de même pour les autres bandes.

IV. Cristaux irradiés, déformés à chaud puis recuits à $400^{\circ} \mathrm{C}$. - N'ayant pas observé, dans la série précédente, de variation significative du coefficient d'absorption, nous avons procédé à une nouvelle série de mesures en recuisant ces cristaux à une température de $400^{\circ} \mathrm{C}$. Cette température avait été choisie, pour se situer peu au-dessous de la temperature de blanchiement total qui se produit en quelques minutes à $450^{\circ} \mathrm{C}$.

Dans une expérience un cristal irrađié avait été déformé en flexion en trois points à $250^{\circ} \mathrm{C}$. Le taux de déformation maximale était de $4 \%$. Après recuit à $400^{\circ} \mathrm{C}$ pendant une demi-heure on constate (Fig. $2 a$ ) que les zones déformées plastiquement sont colorées, alors que le reste du cristal apparaît totalement incolore. Entre polariseurs croisés on peut observer (Fig. $2 b$ ) les plans de glissement dont la localisation coïncide avec celle de la coloration, et avec celle des charges électriques observées en xérographie [6]. Cette coloration est donc en relation avec le mouvement des dislocations.
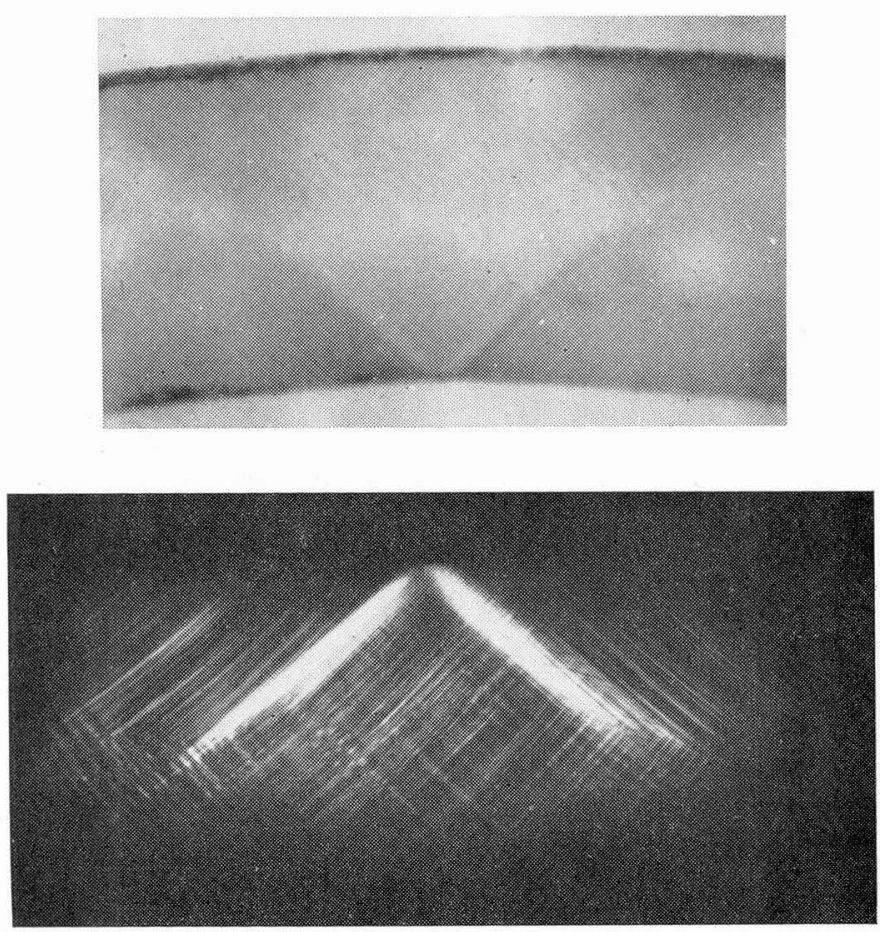

FIG. 2. - a) Cristal de LiF irradić, déformés en flexion à $250^{\circ} \mathrm{C}$, recuit à $400^{\circ} \mathrm{C}$.

b) Le même cristal, entre polariseurs croisés. 
Dans les expériences suivantes, la déformation a été faite à des températures différentes, $150,250,300^{\circ} \mathrm{C}$; le recuit étant toujours effectué à $400^{\circ}$. La figure 3 donne les spectres d'absorption pour deux cristaux de LiF irradiés ayant subi à $300^{\circ} \mathrm{C}$ une déformation de 0,59 et $2 \%$ respectivement et recuits à $400^{\circ} \mathrm{C}$ une demi-heure. Ces spectres ont été faits dans la zone déformée plastiquement; on constate un renforcement très net de la bande $M$; l'absorption correspondant à la bande $F$ augmente aussi, mais de façon moins significative. On remarquera vers $400 \mathrm{~m} \mu$ et vers $480 \mathrm{~m} \mu$ les épaulements $S_{1}$ et $S_{2}$ dont l'origine incertaine pourrait être attribuée soit aux centres $F_{3}^{+}$étudiés par Farge, Toulouse et Lambert [9], soit à un centre d'impuretés.

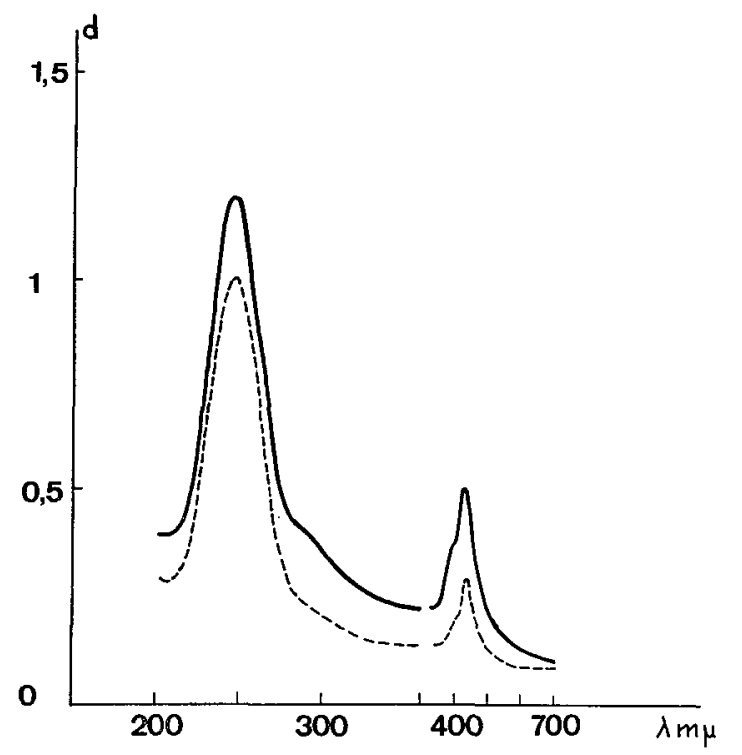

Fig. 3. - Spectres d'absorption de deux cristaux de LiF irradiés déformés en flexion à $300^{\circ} \mathrm{C}$ et recuits à $400^{\circ} \mathrm{C}$. En ordonnée $d$, densité optique. Courbe en tirets : déformation de $0,59 \%$; courbe en trait continu : déformation de $2 \%$. L'interruption dans les courbes correspond à un changement dans l'échelle des abscisses.

Les figures 4 et 5 donnent en fonction du taux de déformation la valeur du coefficient d'absorption pour les différentes températures. On constate tout d'abord sur la figure 4 que pour les centres $M$, le fait que le cristal ait été chauffé d'abord à $150^{\circ}, 250^{\circ}$ ou $300^{\circ} \mathrm{C}$ ne modifie pratiquement pas, pour une déformation nulle, le coefficient d'absorption après recuit à $400^{\circ} \mathrm{C}$. Par contre la persistance des centres $M$ après recuit augmente linéairement avec la déformation, le phénomène étant surtout marqué à $300^{\circ} \mathrm{C}$.

Sur la figure 5 sont reportés les résultats corres-

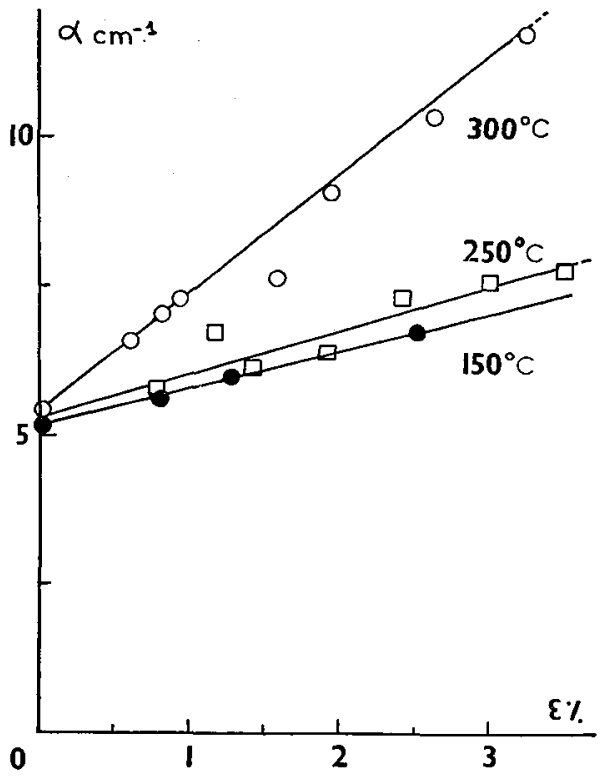

FrG. 4. - Variation du coefficient d'absorption pour la bande $M$, en fonction du taux de déformation maximale. Cristaux déformés à diverses températures et recuits uniformément à $400^{\circ} \mathrm{C}$.

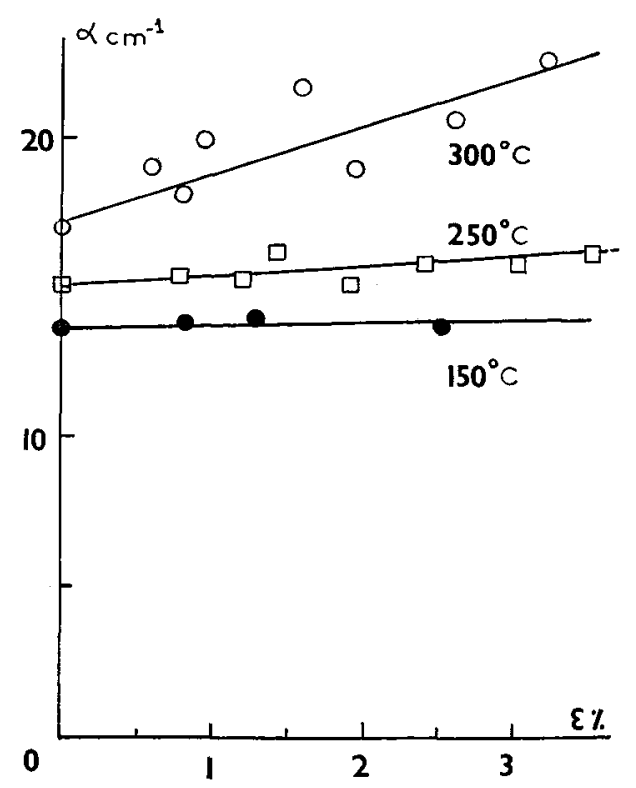

Frg. 5. - Variation du coefficient d'absorption pour la bande $F$. Mêmes cristaux que pour la figure 4 .

pondant à la bande $F$. Les différences qu'on observe pour une déformation nulle, à diverses températures, ne doivent pas être considérées comme significatives, si 
l'on tient compte des erreurs de mesures, l'absorption étant beaucoup plus forte que pour la bande $M$. On trouve, comme pour les centres $M$, une persistance accrue des centres $F$ pour les cristaux déformés. Mais ce fait est moins marqué que pour la bande $M$.

Pour la bande $R_{1}$, ainsi que pour $S_{1}$ et $S_{2}$ on observe de même un persistance accrue avec la déformation.

Partant de cristaux îradiés où l'absorption pour la bande $F$ est de $200 \mathrm{~cm}^{-1}$ environ, après chauffage à $300^{\circ} \mathrm{C}$ pendant une demi-heure sans déformation, suivi d'un recuit à $400^{\circ} \mathrm{C}$ pendant une demj-heure, on trouve une absorption de $17 \mathrm{~cm}^{-1}$. Pour un échantillon ayant subi le même traitement thermique, mais déformé de $3 \%$ maximum alors qu'il était à $300^{\circ}$, on trouve une absorption de $22 \mathrm{~cm}^{-1}$. Bien que la déformation ait augmenté de $30 \%$ le nombre des centres $F$ résiduels, on voit qu'il ne subsiste qu'environ $11 \%$ des centres présents initialement. Pour les centres $M$ l'absorption était initialement de $40 \mathrm{~cm}^{-1}$. La même déformation augmente de $125 \%$ le nombre des centres $M$ résiduels, qui représentent $39 \%$ des centres initiaux.

V. Discussion des résultats expérimentaux. Le premier fait qu'il conviendrait d'expliquer c'est l'augmentation des centres $M$ et $N$ provoquéc par la déformation à température ambiante. Il est vraisembiable que ces centres se forment par transformation d'autres centres; les spectres réalisés ne nous permettent malheureusement que de faire des hypothèses sur leur nature. Dans les cristaux avant déformation on constate la présence de bandes $R_{1}$ et $R_{2}$ assez importantes. Admettons pour les centres $R$ le modele donné par Pick [10]. Au couts du passage des dislocations ces centres peuvent être détruits en donnant naissance à un centre $M$ et à un centre $F$; dans une autre possibilité la dislocation pourtait transporter au moins sur une courte distance un ccn1re $F$, qui venant s'agglomérer au centre $R$, donnerait un centre $N_{2}$. Ces mécanismes peuvent être représcntés par les schémas suivants :

$$
\begin{aligned}
& R \rightarrow F+M \\
& R+F \rightarrow N_{2} .
\end{aligned}
$$

Ces schémas permettraient d'expliquer l'augmentation des bandes $M$ et $N_{2}$ après déformation. Sur la zone déformée, les mesures faites avec le montage microspectrophotometrique, sont limitées au domaine spectral visible, c'est-à-dire que la diminution à prévoir des bandes $R_{1}$ et $R_{2}$ n'a pu être observée. L'anisotropie constatée dans les centres $M$ et $N_{2}$ s'expliquerait soit par l'orientation préfétentielle donnée à ces centres lors de leur formation par un ensemble de dislocations à vecteurs de Burgers paratlèles, soit par une polarisation de ces centres dans le champ des contraintes résiơuelles.

Le recuit des cristaux irradiés augmente la proportion des centres complexes, d'où individualisation des centres $N_{1}$ et $N_{2}$. Au cours de la déformation subséquente, le passage des dislocations peut détruire des centres $N_{1}$ et $N_{2}$. Mais nous avons vu que cette déformation pouvait aussi amener la formation de centres $N_{2}$. Le bilan de l'opération pourra done être une augmentation des centres $N_{2}$ et une diminution des centres $N_{1}$.

En ce qui concerne les cristaux déformés à chaud, il convient de noter que par suite du mode opératoire différent, le taux de la déformation atteint était plus faible que dans les essais faits à froid en compression. C'est probablement ce qui explique l'absence de modification après déformation.

Il reste à interpréter ['influence đu recuit sur les cristaux déformés à chaud : dans les zones déformées tous les défauts semblent persister davantage que dans la zone non déformée. Pour préciser nous avons essayé de calculer le taux de centres piégés par unité de longueur de dislocation, d'une façon analogue à celle que nous avions utilisée pour Tes charges électriques [6]. La densité des dislocations par $\mathrm{cm}^{3}, N d$, peut être estimée à partir de la déformation plastique $\varepsilon_{\mathrm{p}}$ grâce à la formuIe donnée par Johnston et Gilman [11] :

$$
N d=10^{9} \varepsilon_{\mathrm{p}} \text { en } \mathrm{cm} / \mathrm{cm}^{3} .
$$

La densité des centres de couleurs peut se calculer par la formule de Smakula [12]; nous prendrons pour le facteur numérique la valeur $0,87 \times 10^{17}$, correspondant a une forme gaussienne pour la bande d'absorption. Pour les centres $F$ nous adopterons pour la force de l'oscillateur la valeur $f=0,997$. Dans un cristal déformé à $300^{\circ}$ nous avons constaté que la persistance des centres $F$ pour une déformation moyenne de $1,3 \%$ se traduit par rapport au cristal non déformé par une variation du coefficient d'absorption $\Delta x_{F}=3,70 \mathrm{~cm}^{-1}$. On obtient alors pour le nombre de centres $F$ piégés par $\mathrm{cm}$ de dislocation Ia valeur $1,7 \times 10^{9}$. En supposant pour la ligne de dislocation la direction [100], le paramètre sur cette ligne est $4,02 \times 10^{-8} \mathrm{~cm}$. Le nombre de centres $F$ correspondant au paramètre sera done de 68 . D'après les résultats reportés sur les figures 4 et 5 , il existe une relation linéaire entre $\varepsilon_{\mathrm{p}}$ et $\alpha$. Il en résuite que le nombre de centres par unité de dislocations est indépendant du degré de déformation. Le calcul effectué pour les centres $F$ et $M$ à différentes tempsratures donne les résultats suivants : 


\begin{tabular}{|c|c|c|c|c|}
\hline \multirow[b]{2}{*}{$T \cdot C$} & \multicolumn{2}{|c|}{ Centres $F$} & \multicolumn{2}{|c|}{ Centres $M$} \\
\hline & $\begin{array}{l}\text { par cm de } \\
\text { dislocation }\end{array}$ & $\begin{array}{l}\text { par unité } \\
\text { paranè- } \\
\text { trique de } \\
\text { dislocation }\end{array}$ & $\begin{array}{l}\text { par cm de } \\
\text { distocation }\end{array}$ & $\begin{array}{l}\text { par unite } \\
\text { paramé- } \\
\text { trique de } \\
\text { dislocation }\end{array}$ \\
\hline $150^{\circ} \ldots$ & $1,1 \times 10^{9}$ & 44 & $0,5 \times 10^{9}$ & 20 \\
\hline $250^{\circ}$ & $1,1 \times 10^{9}$ & 44 & $0,5 \times 10^{9}$ & 20 \\
\hline $300^{\circ} \ldots$ & $1,7 \times 10^{9}$ & 68 & $1,5 \times 10^{9}$ & 60 \\
\hline
\end{tabular}

Le nombre de centre par unité de dislocation est beaucoup trop élevé pour qu'on puisse supposer que les centres sont situés sur des crans le long de la ligne de dislocation. Par contre il s'explique si on considère que ces centres de type lacunaire se répartissent à I'intérieur de la zone en compression qui est associée à chaque ligne de dislocation. On notera d'ailleurs que les centres complexes, tels $M$ ou $R$, qui corresdondent à un défaut de matière plus important, paraissent davantage attirés que les centres $F$. On peut supposer que sous l'action du champ de contrainte il se produit une ségrégation des défauts. Tandis que ceux de type Iacunaire tendront a migrer vers les parties en compression, ceux de type interstitiel se dirigeront vers les parties en tension. Il en résulte que la destruction des centres au chauffage, qui peut se faire par annihilation d'un défaut de type lacunaire par un défaut de type interstitiel, va voir son processus ralenti dans la zone déformée. Ceci est en accord avec les observations faites sur KCl par Damm [13] qui conclut que les dislocations freinent la diffusion des centres colorés.

\section{Bibliographie}

[1] Wiegand (D. A.), Smoluchowski (R.), Phys, Rev., 1959, 116, p. 1069.

[2] Wiegand (D. A.), Turner (R. G.), Smoluchowski (R.), J. Phys. Chem. Solids, 1966, 27, p. 1251.

[3] Schaeffer (B.), Dupuy (C. H. S.), Saucier (H.), C. R. Acad. Sc. Paris, 1965, 261, p. 5424 .

[4] Dupuy (C. H. S.), Schaeffer (B.), Saucifr (H.), $J$. Physique, 1966, T 27, S. 7, 8, p. C3-21.

[5] Dupuy (C. H. S.), Schaeffer (B.), Proc. British Ceramic Soc., 1966, 6, p. 257.

[6] Dupuy (C. H. S.), Bull. Soc. Franc. Mineral. Cristal., 1965, 88, p. 533.

[7] Kubo (K.), J. Phys. Soc. Jap, 1961, 16, p. 2294.

[8] Van DoORn (C. Z), Havfn (Y.), Phys, Rev, 1955, 100, p. 753 .

[9] Farge (Y.), Toulouse (G.), Lambert (M.), $y$. Physique, $1966,27,5-6$, p. 287.

[10] Pick (H.), Z. Physik, 1960, 159, p. 69.

[11] Johnston (W. G.), Gilman (J. J.), J. Appl. Physics U. S. A., 1959, 30, p. 129.

[12] SMakula (A.), Z. Physik, 1930, 59, p. 603.

[13] Damm (J.Z.), Kowalczyk (J.), Phys. Stat. Sol., 1964 6. p. 693 . 